\title{
Hepatic fat quantification of magnetic resonance imaging whole-liver segmentation for assessing the severity of nonalcoholic fatty liver disease: comparison with a region of interest sampling method
}

\author{
Qin-He Zhang ${ }^{1}$, Ying Zhao ${ }^{1}$, Shi-Feng Tian ${ }^{1}$, Lu-Han Xie ${ }^{2}$, Li-Hua Chen ${ }^{1}$, An-Liang Chen ${ }^{1}$, Nan Wang ${ }^{1}$, \\ Qing-Wei Song ${ }^{1}$, Hao-Nan Zhang ${ }^{1}$, Li-Zhi Xie ${ }^{3}$, Zhi-Wei Shen ${ }^{4}$, Ai-Lian Liu ${ }^{1}$ \\ ${ }^{1}$ Department of Radiology, the First Affiliated Hospital of Dalian Medical University, Dalian, China; ${ }^{2}$ Department of Pathology and Forensics, \\ Dalian Medical University, Dalian, China; ${ }^{3}$ GE Healthcare, MR Research, Beijing, China; ${ }^{4}$ Philips Healthcare, Beijing, China
}

Correspondence to: Ai-Lian Liu. Department of Radiology, the First Affiliated Hospital of Dalian Medical University, Xigang District, 222 Zhongshan Road, Dalian 116011, China. Email: liuailian@dmu.edu.cn.

Background: Accurate and early assessment of the hepatic fat content is crucial for patients with nonalcoholic fatty liver disease (NAFLD). For years, magnetic resonance imaging (MRI) has been considered the optimal noninvasive method for the assessment of fat accumulation. To avoid time-consuming manual placement of multiple regions of interest (ROI), the use of whole-liver segmentation has been proposed to measure liver fat, mainly for heterogeneous fat deposition. However, it remains uncertain whether the hepatic mean fat fraction (FF) obtained by whole-liver segmentation with the inclusion of intrahepatic vasculature is consistent with the traditional ROI sampling method. In this study, we assessed the accuracy of hepatic mean FF obtained by whole-liver segmentation in patients of NAFLD with different severities using the ROI sampling method as a reference standard.

Methods: Hepatic FFs were measured by whole-liver segmentation and the ROI sampling method (reference standard) using MRI scanning with the iterative decomposition of water and fat with echo an asymmetry at least-square estimation-iron quantification (IDEAL-IQ) sequence. SPSS version 25.0 software was used to analyze the correlation and consistency of data between the two methods.

Results: There was a strong correlation in hepatic FF between whole-liver segmentation and the ROI sampling method in healthy, mild, and moderate steatosis patients ( $\mathrm{r}=0.943,0.990$, and 0.961 , respectively). Bland-Altman analysis showed a small bias of $+0.50 \pm 0.27$ and $+0.05 \pm 0.30$, which indicated a small overestimation when using whole-liver segmentation in healthy subjects and mild NAFLD patients. The $95 \%$ limits of agreement ranged from +1.02 to -0.03 , and from +0.65 to -0.55 , respectively. However, a small bias of $-0.96 \pm 0.77$ was also evident, which indicated a small underestimation when using whole-liver segmentation in moderate NAFLD patients. The $95 \%$ limits of agreement ranged from +0.56 to -2.48 .

Conclusions: Due to inclusion of the intrahepatic vasculature, whole-liver segmentation has some effects on hepatic FF assessment in patients with different NAFLD severities; yet, it does not significantly affect the assessment of whole-liver FF in MRI FF maps.

Keywords: Hepatic fat; whole-liver segmentation; nonalcoholic fatty liver disease (NAFLD); magnetic resonance imaging (MRI)

Submitted Aug 21, 2020. Accepted for publication Mar 12, 2021.

doi: 10.21037/qims-20-989

View this article at: http://dx.doi.org/10.21037/qims-20-989 


\section{Introduction}

Nonalcoholic fatty liver disease (NAFLD) is a condition caused by excessive triglyceride accumulation in the liver, which affects approximately $25 \%$ of the global population, mostly overweight or obese people. It is also the most common cause of chronic liver disease. It is closely related to type 2 diabetes mellitus, hypertension, metabolic syndrome, and increased risk of cardiovascular diseases $(1,2)$. If diagnosed early, NAFLD can be successfully cured. Yet, if left untreated, it often results in nonalcoholic steatohepatitis, fibrosis (cirrhosis), and eventually leads to liver failure and even hepatic cell cancer (3). Therefore, accurate and early assessment of the hepatic fat content is crucial for patients with NAFLD.

Liver biopsy is considered the gold standard for assessing hepatic fat fraction (FF) that reveals a semiquantitative analysis of NAFLD; yet, it is an invasive approach that is not universally accepted by patients. Furthermore, only a small part of the liver is collected by biopsy, which may lead to sampling error, especially in patients with diffuse hepatic steatosis, which tends to be inhomogeneous throughout the liver (4).

A magnetic resonance imaging (MRI) iterative decomposition of water and fat with an echo asymmetry at least-square estimation-iron quantification (IDEALIQ) sequence can be used to accurately quantify hepatic fat deposition (5). This technique uses a 6-echo 3-point Dixon method to minimize $\mathrm{T} 2 *$ interference. It reduces the effects of fat peak multiplicity, signal-to-noise ratio deviation, and eddy currents on the results, making proton density in tissues the main factor affecting signal intensity (6). By using IDEAL-IQ, accurate and reliable whole hepatic FF can be automatically obtained without postprocessing or calculation by a single breath-hold scanning. A recent meta-analysis showed that IDEAL-IQ has high diagnostic accuracy in detecting and grading hepatic steatosis with histology as the reference standard, suggesting that IDEALIQ can provide accurate quantification of hepatic steatosis in clinical trials and patient care (7). However, so far, no standards have been developed for using the hepatic FF region of interest (ROI) sampling in FF maps.

Hepatic steatosis tends to diffuse across the liver; however, its distribution may be uneven. Due to spatial heterogeneity in hepatic fat deposition, differences in the ROI sampling method can lead to fat quantification variability. Generally, sampling liver segments in both lobes and including a total surface area equal to or larger than $5 \mathrm{~cm}^{2}$ are used to provide a close estimate of the liver mean FF $(1,8)$; however, this method is not easy to perform. To avoid time-consuming manual placement of multiple ROIs, the use of whole-liver segmentation has been proposed to assess liver fat, mainly if the fat deposition is heterogeneous (9-11). Still, it remains uncertain whether the hepatic mean FF obtained by wholeliver segmentation due to the inclusion of intrahepatic vasculature is consistent with traditional ROI sampling methods.

The aim of this study was to assess the accuracy of hepatic mean FF obtained by whole-liver segmentation in patients with different severities of NAFLD using the traditional ROI sampling method as a reference standard.

\section{Methods}

\section{Study population}

This single-center, retrospective study included 1,818 patients who underwent upper abdominal MRI examination between January 2017 and December 2019. Of these patients, those who fulfilled at least 1 of the following criteria were excluded: age $<18$ years $(n=8)$; a history of heavy drinking (alcohol consumption $\geq 30 \mathrm{~g}$ per week in men or $\geq 20 \mathrm{~g}$ per week in women in the last 10 years) $(\mathrm{n}=72)$; any clinical evidence of secondary causes of hepatic steatosis $(\mathrm{n}=73)$; evidence of other liver diseases $(\mathrm{n}=154)$, including viral hepatitis, autoimmune liver diseases, drug-induced liver injury, and so on; evidence of cirrhosis, malignant liver tumor, benign liver tumor (larger than two-thirds of each liver segment), liver posthepatectomy, decompensated liver diseases $(n=984)$; no IDEAL-IQ sequence $(n=128)$; poor image quality (poor signal-to-noise ratio or motion artifacts) $(\mathrm{n}=6)$; intrahepatic bile duct dilation $(\mathrm{n}=44)$. Finally, 349 participants (159 men and 190 women) were included in the study.

The local ethical committee approved the study, and the written consent was provided by all participants.

\section{MRI examinations}

In this study, an MRI scanner (GE Medical Systems, Inc., Waukesha, WI, USA) with an 8-channel phased-array body coil was used. Before scanning, patients fasted for 4 6 hours and were trained to exhale and hold their breath for more than 20 seconds. The participants were examined in the supine position. A 3-plane localization imaging gradient-echo sequence was performed at the beginning of 

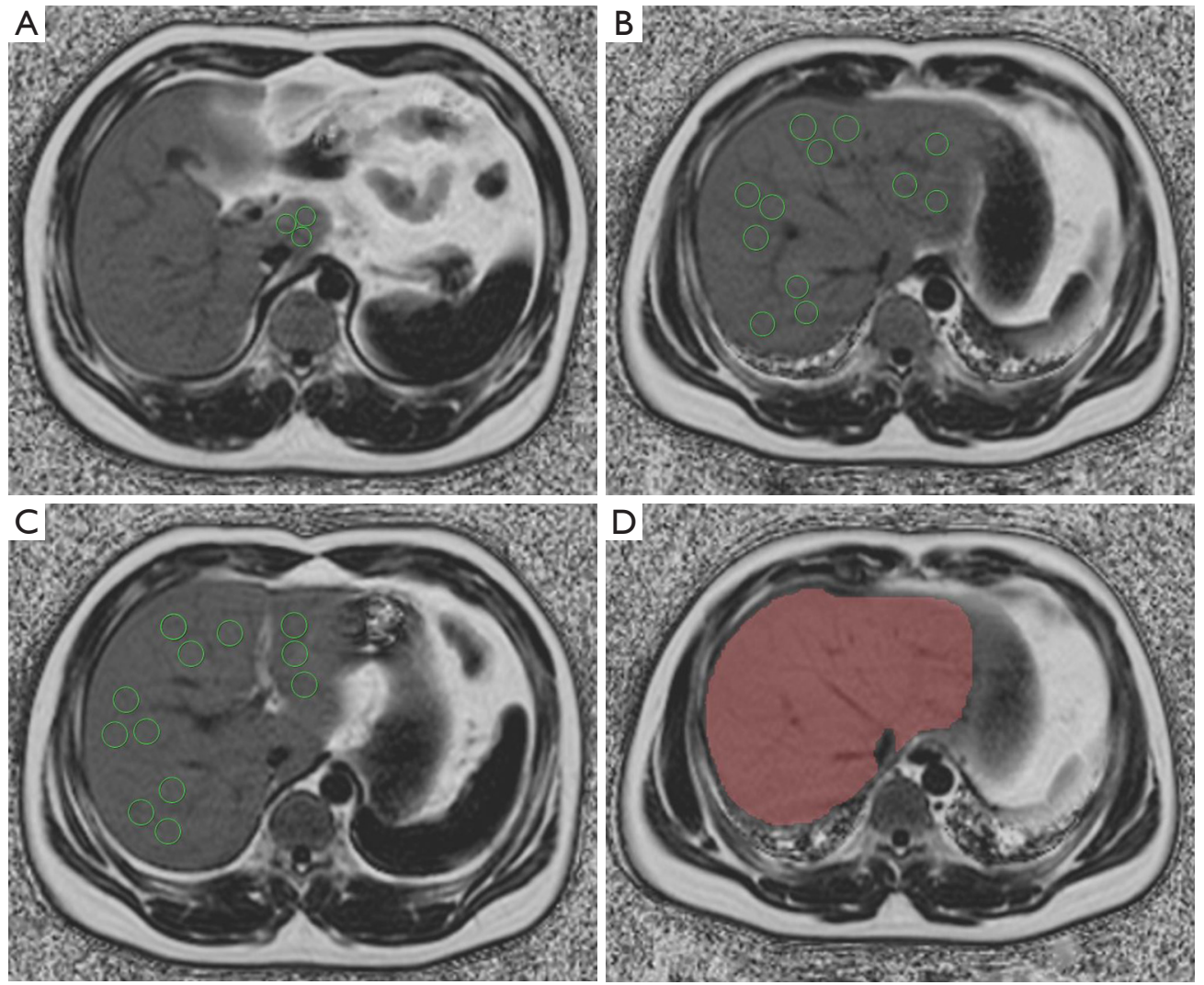

Figure 1 Hepatic FF measurement with the ROI sampling method (A,B,C) and whole-liver segmentation (D) in a 27-year-old man. We placed 3 ROIs in each of the 9 anatomical Couinaud liver segments (27 separate ROIs) while avoiding major vessels, bile ducts, and liver edges. The whole hepatic tissue was semiautomatically traced on FF maps. The hepatic FFs (\%) were 29.7 and 27.7, respectively. FF, fat fraction; ROI, region of interest.

acquisition.

The IDEAL-IQ sequence and routine MRI Ax T1 fast spoiled gradient-recalled (FSPGR), Ax T2 fast-spine echo (FSE), diffusion-weighted imaging (DWI) sequence, and dual echo sequence were acquired. The MRI parameters were as follows: 3.0 T MRI IDEAL-IQ sequence: repetition time/time to echo $(\mathrm{TR} / \mathrm{TE})=6.9 \mathrm{~ms} / 3.0 \mathrm{~ms}$, slice thickness $=10 \mathrm{~mm}$, bandwidth $=200 \mathrm{KHz}$, field of view $(\mathrm{FOV})=36 \mathrm{~cm}$ $\times 36 \mathrm{~cm}$, matrix $=256 \times 160$, flip angle $=3^{\circ}$, number of excitation $(\mathrm{NEX})=1$, breath holding for less than $24 \mathrm{~s}$; 1.5 T MRI IDEAL-IQ sequence: TR/TE $=13.4 \mathrm{~ms} / 4.8 \mathrm{~ms}$, slice thickness $=10 \mathrm{~mm}$, bandwidth $=125 \mathrm{KHz}, \mathrm{FOV}=36 \mathrm{~cm}$ $\times 36 \mathrm{~cm}$, matrix $=256 \times 160$, flip angle $=5^{\circ}, \mathrm{NEX}=1$, breath holding for less than $24 \mathrm{~s}$; T1WI sequence: TR/TE $=$ $210 \mathrm{~ms} / 2.4 \mathrm{~ms}$; T2WI: TR/TE $=8,571 \mathrm{~ms} / 100 \mathrm{~ms}$; dual echo sequence: $\mathrm{TR}=190 \mathrm{~ms}$, TE $=2,4.3 \mathrm{~ms}$; DWI sequence: $\mathrm{TR} / \mathrm{TE}=7,500 \mathrm{~ms} / 58 \mathrm{~ms}, \mathrm{NEX}=4$, $\mathrm{b}$ value $=0,600 \mathrm{~s} / \mathrm{mm}^{2}, \mathrm{FOV}=42 \mathrm{~cm} \times 42 \mathrm{~cm}$. The images were processed using IDEAL Research software provided by the manufacturer (GE Healthcare, Waukesha, WI, USA) to generate water-phase, fat-phase, in-phase, out-phase, along with $\mathrm{R} 2 *$ and $\mathrm{FF}$ maps.

\section{ROI sampling methods}

Hepatic FF was manually calculated using a circular ROI on the FF maps in an open-source vendor-neutral postprocessing platform (ISP, Philips Interspace Portal, Philips Healthcare, Amsterdam, Netherlands), and blood vessels, bile ducts, liver boundaries, and focal hepatic lesions were avoided. A total of 3 ROIs were placed in each of the 9 anatomical Couinaud liver segments (27 separate ROIs) (Figure 1A,B,C). Hepatic FFs in 27 ROIs were averaged to provide the mean hepatic FF. The hepatic FF protocol and ROI sampling method were based on a previous study (1). Similar to the previous study performed in Chinese populations (12), participants were 
categorized into 4 groups: healthy (hepatic FF <5\%), mild $(5 \% \leq$ hepatic $\mathrm{FF} \leq 14 \%)$, moderate $(14 \%<$ hepatic $\mathrm{FF} \leq 28 \%)$, or severe (hepatic FF > 28\%) steatosis.

\section{Whole-liver segmentation}

On the image signal processor (ISP) post-processing platform, the software algorithm (multimodality tumor tracking, MMTT) defined the liver's margins in 3 dimensions, and the whole hepatic tissue was semiautomatically traced on FF maps. If the margins needed tweaking, the operator made corrections; if margins were included within the liver segmentation contours, the main portal vein, inferior vena cava, and the gallbladder were manually removed. Then, hepatic tissue was segmented, and the whole hepatic FF was automatically calculated (Figure 1D).

\section{Inter- and intraobserver variability}

A total of 43 patients were selected between January 2017 and April 2017, and the data were measured independently by radiologists $\mathrm{A}$ and $\mathrm{B}$ (with 10 and 7 years of experience in abdominal imaging MR diagnosis, respectively). A week later, radiologist A carried out a second measurement of the same images to evaluate intraobserver data's repeatability. Finally, radiologist A completed the remaining data measurement. Both radiologists were blinded to the grouping.

\section{Statistical analysis}

All data were analyzed using SPSS version 25.0 (IBM Corp., Armonk, NY, USA). The Shapiro-Wilk test was used to test the normality of the variables. Normally distributed data are expressed as means \pm standard deviations, and nonnormally distributed data are expressed as medians and ranges (25th, 75th percentiles). Nominal data are expressed as a percentage. The intraclass correlation coefficient (ICC) was used to check the 2 observers' consistency: ICC value $<0.4$ indicated poor consistency, while ICC value $>0.75$ indicated good consistency. Bland-Altman plots assessed the consistency between different methods. When parameters were normally distributed, Pearson's correlation coefficient was used; otherwise, Spearman's correlation coefficient was applied. Correlation coefficients were interpreted as follows: weak, 0-0.4; moderate, 0.4-0.7; strong, 0.7-1.0. Scatter plots were generated to visualize the correlation between hepatic FF of different methods. Furthermore, absolute differences for hepatic FF were calculated. A two-tailed $\mathrm{P}$ value $<0.05$ was considered statistically significant.

\section{Results}

A total of 349 patients were included in the study. The participants' age, gender, height, weight, and body mass index (BMI) are shown in Table 1. Regular liver fat content was found in 223 participants $(63.90 \%)$, whereas 97 (27.79\%), 27 (7.74\%), and 2 participants (0.57\%) had mild, moderate, or severe steatosis, respectively.

\section{Consistency analysis}

The data consistency is shown in Table 2. The intraobserver and interobserver ICC values in whole-liver segmentation were 0.998 and 0.999 , respectively. The intraobserver and interobserver ICC values in the ROI sampling method were 0.998 and 0.999 , respectively. All ICC values were $>0.9$, which indicated good interobserver and intraobserver agreement.

\section{Correlation and Bland-Altman analysis}

The hepatic FF (\%) of whole-liver segmentation and the ROI sampling method in healthy participants were 3.10 $(2.50,3.90)$ and $2.56(2.01,3.28)$ respectively, and a strong correlation in hepatic FF was found between the two methods ( $\mathrm{r}=0.943, \mathrm{P}<0.001)$. The hepatic FF (\%) of wholeliver segmentation and the ROI sampling method in mild steatosis patients was $7.80(6.30,10.00)$ and 7.71 (6.25, $10.00)$, respectively, which showed a strong correlation in hepatic FF between the two methods ( $\mathrm{r}=0.990, \mathrm{P}<0.001)$. The hepatic FF (\%) of whole-liver segmentation and the ROI sampling method in moderate steatosis patients was $17.00(14.30,22.37)$ and $18.03(15.15,22.37)$, respectively, which showed a strong correlation in hepatic FF between two methods $(\mathrm{r}=0.961, \mathrm{P}<0.001)$ (Figure 2). The detailed measurements are shown in Table 3.

Bland-Altman analysis showed a small bias of $+0.50 \pm 0.27$ and $+0.05 \pm 0.30$, which indicated a small overestimation by semiautomatic segmentation in healthy participants and mild NAFLD patients, respectively. The $95 \%$ limits of agreement ranged from +1.02 to -0.03 , and from +0.65 to -0.55 , respectively. However, a small bias of $-0.96 \pm 0.77$ was revealed, which indicated a small underestimation by semiautomatic segmentation in moderate NAFLD patients. The $95 \%$ limits of agreement 
Table 1 General characteristics of the study population

\begin{tabular}{|c|c|c|c|}
\hline Parameter & $\begin{array}{c}\text { Healthy } \\
\text { (mean hepatic FF, <5\%) }\end{array}$ & $\begin{array}{c}\text { Mild steatosis } \\
\text { (mean hepatic FF, 5-14\%) }\end{array}$ & $\begin{array}{c}\text { Moderate steatosis } \\
\text { (mean hepatic FF, }>14 \% \text { ) }\end{array}$ \\
\hline No. of participants & 223 & 97 & 29 \\
\hline \multicolumn{4}{|l|}{ Sex } \\
\hline Male & 195 & 51 & 13 \\
\hline Age, years & $58.00(50.00,66.00)$ & $57.24 \pm 12.07$ & $53.00 \pm 14.28$ \\
\hline Weight, kg & $67.00(60.00,75.00)$ & $75.12 \pm 11.34$ & $78.71 \pm 13.15$ \\
\hline Height, m & $1.65(1.60,1.74)$ & $1.70(1.62,1.75)$ & $1.68 \pm 0.12$ \\
\hline BMI, $\mathrm{kg} / \mathrm{m}^{2}$ & $23.83(21.96,26.33)$ & $26.43 \pm 2.51$ & $27.81 \pm 2.31$ \\
\hline Diabetes, n (\%) & $0(0)$ & $27(27.84)$ & $29(100.00)$ \\
\hline Antihypertensive treatment, $\mathrm{n}(\%)$ & $68(30.49)$ & 38 (39.18) & $9(31.03)$ \\
\hline Diabetes treatment, n (\%) & $0(0)$ & $14(14.43)$ & $29(100.00)$ \\
\hline Alcohol use, n (\%) & $13(5.83)$ & $2(2.06)$ & $1(3.45)$ \\
\hline Current smoking, n (\%) & $24(10.76)$ & $2(2.06)$ & $2(6.90)$ \\
\hline
\end{tabular}

*, missing 1 participant's data. FF, fat fraction; BMI, body mass index; ALT, alanine aminotransferase; AST, aspartate aminotransferase.

Table 2 Measurement consistency of two observers

\begin{tabular}{|c|c|c|c|c|c|c|}
\hline Methods & $\mathrm{N}$ & Radiologist A1 & Radiologist A2 & $\begin{array}{c}\text { ICC value } \\
\text { (intraobserver) }\end{array}$ & Radiologist B & $\begin{array}{c}\text { ICC value } \\
\text { (interobserver) }\end{array}$ \\
\hline $\begin{array}{l}\text { Hepatic FF of whole-liver } \\
\text { segmentation (\%) }\end{array}$ & 43 & $4.10(2.85,6.90)$ & $3.90(2.65,7.10)$ & 0.998 & $3.90(2.70,7.15)$ & 0.999 \\
\hline $\begin{array}{l}\text { Hepatic FF of ROI sampling } \\
\text { method (\%) }\end{array}$ & 43 & $3.36(2.31,6.79)$ & $3.73(2.51,6.58)$ & 0.998 & $3.63(2.62,6.67)$ & 0.999 \\
\hline
\end{tabular}

ICC, the intraclass correlation coefficient; FF, fat fraction; ROI, region of interest.

ranged from +0.56 to -2.48 (Figure 3 ).

\section{The over-and underestimation of the degree of hepatic steatosis of whole-liver segmentation}

As shown in Table 4, the number of healthy, mild, and moderate steatosis participants were 223, 97, and 29, respectively, according to the traditional ROI sampling method, and the number of healthy, mild, and moderate steatosis participants were 213,110 , and 26 according to whole-liver segmentation. Thus, 10 healthy participants were incorrectly classified into the mild steatosis group, while 3 moderate steatosis patients were incorrectly assigned to the mild fatty liver group.

\section{Discussion}

This study evaluated the accuracy of hepatic FF using whole-liver segmentation compared to the traditional ROI sampling method. Our results revealed an excellent agreement and correlation between the two methods. There was a slight overestimation of hepatic fat content 

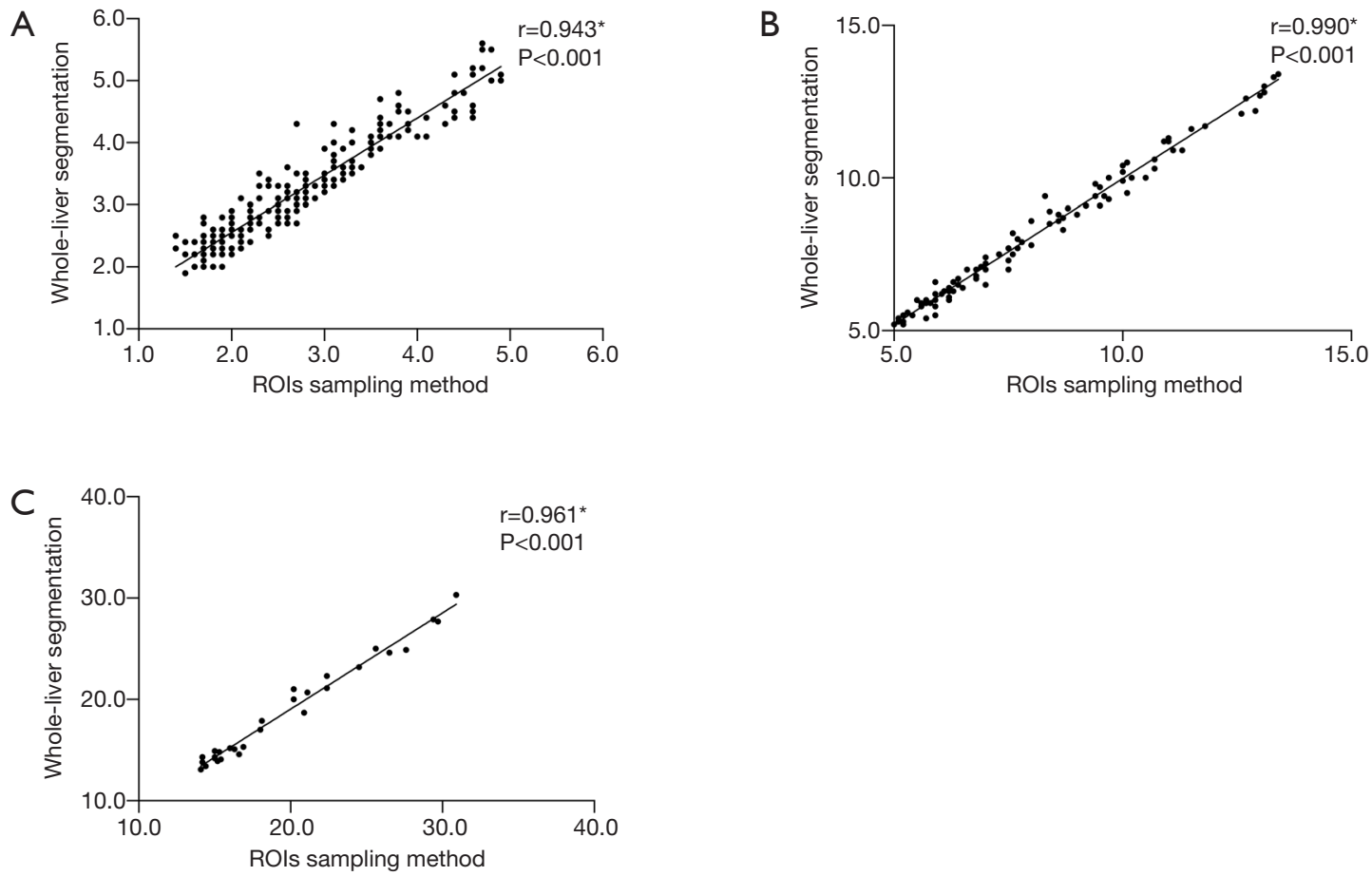

Figure 2 The correlation in hepatic FF between whole-liver segmentation and ROI sampling method. (A) Healthy subjects; (B) mild NAFLD; (C) moderate NAFLD. *, Spearman's correlation coefficient. FF, fat fraction; ROI, region of interest; NAFLD, nonalcoholic fatty liver disease.

Table 3 Correlation of whole-liver segmentation and the ROI sampling method

\begin{tabular}{|c|c|c|c|c|}
\hline Hepatic steatosis severity & HFF of whole-liver segmentation (\%) & HFF of ROI sampling method (\%) & $r$ & $P$ value \\
\hline Mild $(n=97)$ & $7.80(6.30,10.00)$ & $7.71(6.25,10.00)$ & 0.990 & $<0.001$ \\
\hline Moderate $(n=29)$ & $17.00(14.30,22.37)$ & $18.03(15.15,22.37)$ & 0.961 & $<0.001$ \\
\hline
\end{tabular}

$\mathrm{ROI}$, region of interest; HFF, hepatic fat fraction.

in healthy people and mild NAFLD patients using wholeliver segmentation [bias $(\%)=+0.51 \pm 0.27$ and $+0.05 \pm 0.30$, respectively]. However, hepatic fat content was slightly underestimated in moderate NAFLD patients [bias (\%) $=-0.96 \pm 0.77]$. In our study, 10 healthy participants were incorrectly classified into the mild steatosis group, and 3 moderate steatosis patients were incorrectly assigned to the mild fatty liver group.

For years, MRI has been considered the optimal noninvasive method for the assessment of fat accumulation. Previous studies have quantified the FF by chemical shift imaging (CSI) technology using a declining degree of MR signal on the out-phase image $(12,13)$; still, its accuracy has been affected by multiple factors, including T1 value, T2 value, and heterogeneity of fat proton. As a gold standard approach of fat quantification, MR spectroscopy (MRS) provides high safety and accuracy for hepatic fat quantification (14); when the fat content is low, its sensitivity is high. Nevertheless, the traditional MRS requires long postprocessing time, patient respiratory coordination, and sampling errors associated with a low spatial resolution. In addition, skill and knowledge are necessary for its accurate performance and spectroscopy data analysis (12). The MRI IDEAL-IQ has an advantage in quantifying liver fat content compared to MRS. With a low flip angle to suppress the $\mathrm{T} 1$ effects and multi-echo acquisition correction of the 
A

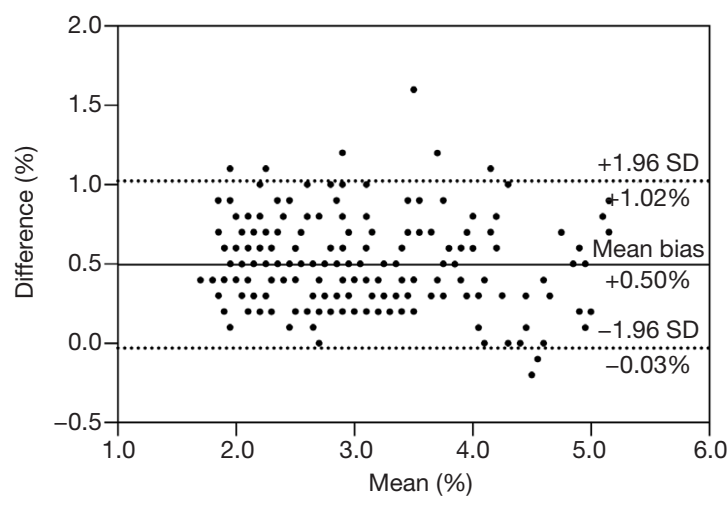

C

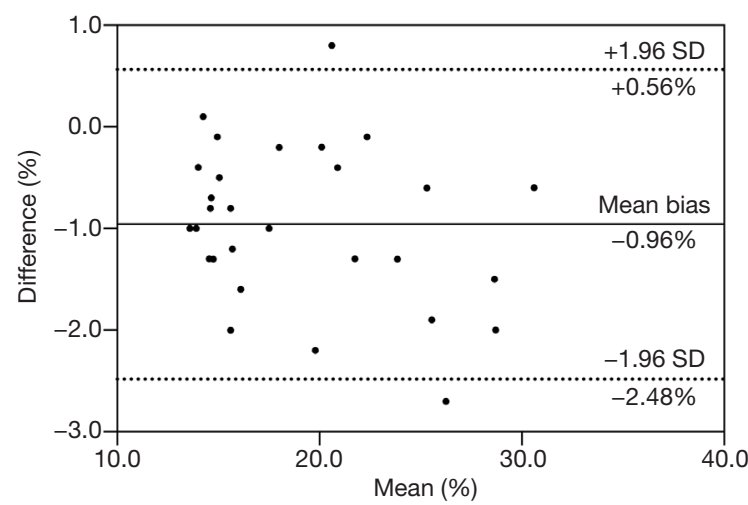

B

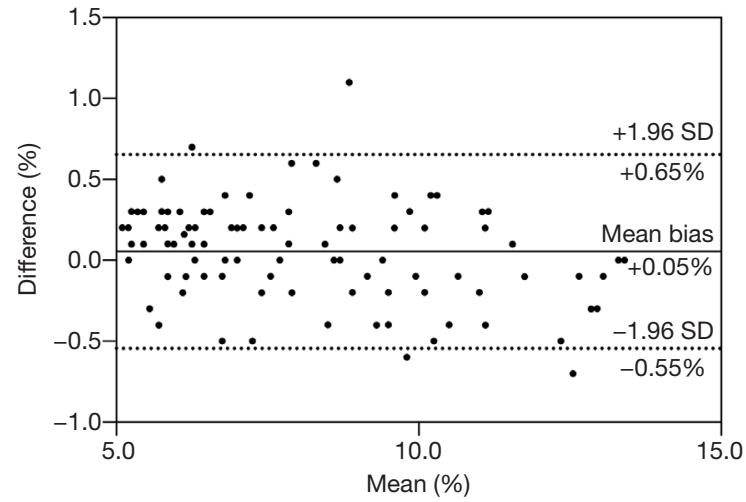

Figure 3 Bland-Altman plots. (A) Bland-Altman plot showing a bias of $+0.50 \%$ with a $95 \%$ limit of agreement ranging from $+1.02 \%$ to $-0.03 \%$ in healthy participants. (B) Bland-Altman plot showing a bias of $+0.05 \%$ with a $95 \%$ limit of agreement ranging from $+0.65 \%$ to $-0.55 \%$ in mild NAFLD. (C) Bland-Altman plot showing a bias of $-0.96 \%$ with a $95 \%$ limit of agreement ranging from $+0.56 \%$ to $-2.48 \%$ in moderate NAFLD. NAFLD, nonalcoholic fatty liver disease.

Table 4 Steatosis categorization by using whole-liver segmentation versus traditional ROI sampling method

\begin{tabular}{lcccc}
\hline \multirow{2}{*}{$\begin{array}{l}\text { Whole-liver } \\
\text { segmentation }\end{array}$} & \multicolumn{2}{c}{ Traditional ROI sampling method } & \multirow{2}{*}{ Total } \\
\cline { 2 - 4 } & Healthy & Mild & Moderate & \\
\hline Healthy & 213 & 0 & 0 & 213 \\
Mild & 10 & 97 & 3 & 110 \\
Moderate & 0 & 0 & 26 & 26 \\
Total & 223 & 97 & 29 & 349 \\
\hline
\end{tabular}

ROI, region of interest.

T2 effects, the IDEAL-IQ sequence offers more accurate modeling for measuring triglyceride fat content (15). Serai et al. (16) suggested that the estimation of hepatic FF with MRI using multipoint Dixon techniques is highly reproducible across readers, field strengths, and imaging platforms. Thus, in the present study, data in 2 different field strengths were analyzed together.

Typically, hepatic FF estimation is obtained by ROI sampling methods, which include single-ROI $(17,18)$, multiple-ROI sampling of the right hepatic lobe $(19,20)$, and multiple-ROI sampling of both lobes (21-23). Even so, an uneven spatial distribution of liver fat may lead to an inaccurate assessment of FF. Previous studies have observed lower FF in the left hepatic lobe and higher FF in the right lobe, attributed to preferential shunting of fatty mesenteric blood toward the right portal vein and shunting of splenic blood toward the left portal vein $(12,21)$. Therefore, ROI location may affect liver mean FF estimation, which may be overestimated when the right lobe is exclusively or predominantly sampled. It has been recommended that hepatic fat content quantification based on MRI FF maps should sample each liver segment and include a total surface area equal or larger than $5 \mathrm{~cm}^{2}$ to provide a close estimate 
of the hepatic mean FF (1).

The estimation of hepatic mean FF requires wholeliver segmentation, especially automated or semiautomated segmentation methods. These approaches not only overcome sampling errors due to steatosis heterogeneity but also save time (24). Stocker et al. (25) evaluated the performance of an automated workflow of hepatic FF with automated inline liver volume segmentation. This approach was proven to be very useful for patients with and without hepatic steatosis. Yet, in cases with iron overload and posthemihepatectomy, extrahepatic areas were erroneously included to a greater extent, resulting in the overestimation of hepatic FF.

In this study, we observed excellent agreement and correlation between whole-liver segmentation and ROI sampling methods in different NAFLD patient grades. Our data indicated that inclusion of the intrahepatic vasculature with the whole-liver segmentation method had a slight effect on assessing mean hepatic FF, which caused a minor overestimation in healthy and mild hepatic steatosis patients, and a minor underestimation in moderate hepatic steatosis patients. However, it did not meaningfully impact the assessment of whole-liver FF in MRI FF maps, thus suggesting that whole-liver segmentation methods for assessing hepatic FF in MRI FF maps may not be necessary to exclude intrahepatic vasculature. Our results were similar to those of $\mathrm{Vu}$ et al., who reported that whole-liver segmentation was correlated with traditional ROI sampling methods $(\mathrm{r}=0.975)$ in a cohort of 35 patients with a mean hepatic FF of 2.1-32.5\% (8). In addition, Kim et al. (11) evaluated the hepatic fat deposition using a free-drawn ROI measurement, which covered nearly the entire liver at 3 different levels (including the confluence of the right hepatic vein, the umbilical portion of the left portal vein, and the posterior branch of the right portal vein) along the liver margin in 156 patients (54 steatosis patients and 102 non-steatosis patients). They assumed that this method could be considered a new reference standard for MR fat quantification instead of MRS. In our study, participants were divided into different groups according to the degree of hepatic steatosis to accurately evaluate the degree of over and underestimation of hepatic steatosis of wholeliver segmentation in a larger sample size. Considering there have been no studies on hepatic steatosis assessment of whole-liver segmentation in FF map with a large patient cohort, our results showed that this method enables accurate hepatic fat quantification.

Our results also showed that 10 healthy participants were incorrectly classified into the mild steatosis group, while 3 moderate steatosis patients were incorrectly assigned to the mild fatty liver group. This suggested that when the liver fat content is near the critical value of mild and moderate hepatic steatosis, the two methods should be used to avoid over- and underestimating the degree of hepatic steatosis.

This study had several limitations. First, this was a retrospective study with a small sample size (particularly for patients with moderate or severe NAFLD), which might have generated some bias. Only 2 participants had severe steatosis, so the accuracy of whole-liver segmentation to evaluate severe steatosis was not analyzed separately. Our results should be further verified by a large-scale prospective validation study in a more general population. Second, we did not correlate our hepatic FF with histopathology or MRS as the standard of reference because the assessment of diagnostic accuracy of IDEAL-IQ sequence was not within the range of this study and had been previously validated. Moreover, biopsy and MRS samples included only a small fraction of the liver volume and might have led to sampling errors. Third, the study was limited to the use of software and a concrete MR scanner (GE Medical Systems). Finally, we did not compare the time spent by the analysts using the two different methods.

In conclusion, our findings demonstrated that the wholeliver segmentation method has some effect on assessing hepatic FF in different severities of NAFLD due to the inclusion of the intrahepatic vasculature, which does not significantly impact whole-liver FF assessment in MRI FF maps. These data suggest that whole-liver segmentation of assessing hepatic FF in MRI FF maps may not be necessary to exclude intrahepatic vasculature.

\section{Acknowledgments}

Funding: This work was supported by Program for Training Capital Science and Technology Leading Talents (Z181100006318003).

\section{Footnote}

Conflicts of Interest: All authors have completed the ICMJE uniform disclosure form (available at http://dx.doi. org/10.21037/qims-20-989). The authors have no conflicts of interest to declare.

Ethical Statement: The local ethical committee approved the study, and the written consent was provided by all 
participants.

Open Access Statement: This is an Open Access article distributed in accordance with the Creative Commons Attribution-NonCommercial-NoDerivs 4.0 International License (CC BY-NC-ND 4.0), which permits the noncommercial replication and distribution of the article with the strict proviso that no changes or edits are made and the original work is properly cited (including links to both the formal publication through the relevant DOI and the license). See: https://creativecommons.org/licenses/by-nc-nd/4.0/.

\section{References}

1. Chen J, Duan S, Ma J, Wang R, Chen J, Liu X, Xue L, Xie S, Yao S. MRI-determined liver fat correlates with risk of metabolic syndrome in patients with nonalcoholic fatty liver disease. Eur J Gastroenterol Hepatol 2020;32:754-61.

2. Mantovani A, Byrne CD, Bonora E, Targher G. Nonalcoholic Fatty Liver Disease and Risk of Incident Type 2 Diabetes: A Meta-analysis. Diabetes Care 2018;41:372-82.

3. Lauridsen BK, Stender S, Kristensen TS, Kofoed KF, Køber L, Nordestgaard BG, Tybjærg-Hansen A. Liver fat content, non-alcoholic fatty liver disease, and ischaemic heart disease: Mendelian randomization and meta-analysis of 279013 individuals. Eur Heart J 2018;39:385-93.

4. Ratziu V, Charlotte F, Heurtier A, Gombert S, Giral P, Bruckert E, Grimaldi A, Capron F, Poynard T; LIDO Study Group. Sampling variability of liver biopsy in nonalcoholic fatty liver disease. Gastroenterology 2005;128:1898-906.

5. Idilman IS, Aniktar H, Idilman R, et al. Hepatic steatosis: quantification by proton density fat fraction with MR imaging versus liver biopsy. Radiology 2013;267:767-75.

6. Wang Q, Ye F, Ma P, Chen F, Che Y, Zhao X, Yang L. Quantitative magnetic resonance imaging evaluation of hepatic fat content with iron deposition: will it be disturbed? J Int Med Res 2019;47:1958-74.

7. Qu Y, Li M, Hamilton G, Zhang YN, Song B. Diagnostic accuracy of hepatic proton density fat fraction measured by magnetic resonance imaging for the evaluation of liver steatosis with histology as reference standard: a metaanalysis. Eur Radiol 2019;29:5180-9.

8. Vu KN, Gilbert G, Chalut M, Chagnon M, Chartrand G, Tang A. MRI-determined liver proton density fat fraction, with MRS validation: Comparison of regions of interest sampling methods in patients with type 2 diabetes. J Magn
Reson Imaging 2016;43:1090-9.

9. Pirimoglu B, Sade R, Islek A, Kantarci M. The Liver Fat Fraction and Abdominal Subcutaneous and Visceral Fat Volume Distribution in Normal-Weight, Overweight, and Obese Children Using a New Magnetic Resonance Imaging Technique. J Comput Assist Tomogr 2019;43:194-9.

10. Graffy PM, Sandfort V, Summers RM, Pickhardt PJ. Automated Liver Fat Quantification at Nonenhanced Abdominal CT for Population-based Steatosis Assessment. Radiology 2019;293:334-42.

11. Kim KY, Song JS, Kannengiesser S, Han YM. Hepatic fat quantification using the proton density fat fraction (PDFF): utility of free-drawn-PDFF with a large coverage area. Radiol Med 2015;120:1083-93.

12. Kang BK, Kim M, Song SY, Jun DW, Jang K. Feasibility of modified Dixon MRI techniques for hepatic fat quantification in hepatic disorders: validation with MRS and histology. Br J Radiol 2018;91:20170378.

13. Nadarajah C, Fananapazir G, Cui E, Gichoya J, Thayalan $\mathrm{N}$, Asare-Sawiri M, Menias CO, Sandrasegaran K. Association of pancreatic fat content with type II diabetes mellitus. Clin Radiol 2020;75:51-6.

14. Livingstone RS, Begovatz P, Kahl S, Nowotny B, Strassburger K, Giani G, Bunke J, Roden M, Hwang JH. Initial clinical application of modified Dixon with flexible echo times: hepatic and pancreatic fat assessments in comparison with (1)H MRS. MAGMA 2014;27:397-405.

15. Hijona E, Sánchez-González J, Alústiza JM, Hijona L, Arenas J, García E, Rojas N, Portillo MP, Jiménez R, Aldazabal P, Bujanda L. Accurate fat fraction quantification by multiecho gradient-recalled-echo magnetic resonance at $1.5 \mathrm{~T}$ in rats with nonalcoholic fatty liver disease. Eur J Radiol 2012;81:1122-7.

16. Serai SD, Dillman JR, Trout AT. Proton Density Fat Fraction Measurements at 1.5- and 3-T Hepatic MR Imaging: Same-Day Agreement among Readers and across Two Imager Manufacturers. Radiology 2017;284:244-54.

17. Yokoo T, Shiehmorteza M, Hamilton G, Wolfson T, Schroeder ME, Middleton MS, Bydder M, Gamst AC, Kono Y, Kuo A, Patton HM, Horgan S, Lavine JE, Schwimmer JB, Sirlin CB. Estimation of hepatic protondensity fat fraction by using MR imaging at $3.0 \mathrm{~T}$. Radiology 2011;258:749-59.

18. Meisamy S, Hines CD, Hamilton G, Sirlin CB, McKenzie CA, Yu H, Brittain JH, Reeder SB. Quantification of hepatic steatosis with T1-independent, T2-corrected MR imaging with spectral modeling of fat: blinded comparison 
with MR spectroscopy. Radiology 2011;258:767-75.

19. Lee SS, Park SH, Kim HJ, Kim SY, Kim MY, Kim DY, Suh DJ, Kim KM, Bae MH, Lee JY, Lee SG, Yu ES.

Non-invasive assessment of hepatic steatosis: prospective comparison of the accuracy of imaging examinations. J Hepatol 2010;52:579-85.

20. Hwang I, Lee JM, Lee KB, Yoon JH, Kiefer B, Han JK, Choi BI. Hepatic steatosis in living liver donor candidates: preoperative assessment by using breath-hold tripleecho MR imaging and $1 \mathrm{H}$ MR spectroscopy. Radiology 2014;271:730-8.

21. Bonekamp S, Tang A, Mashhood A, Wolfson T, Changchien C, Middleton MS, Clark L, Gamst A, Loomba R, Sirlin CB. Spatial distribution of MRIDetermined hepatic proton density fat fraction in adults with nonalcoholic fatty liver disease. J Magn Reson Imaging 2014;39:1525-32.

22. Hu F, Yang R, Huang Z, Wang M, Yuan F, Xia C, Wei Y, Song B. 3D Multi-Echo Dixon technique for simultaneous assessment of liver steatosis and iron overload in patients with chronic liver diseases: a feasibility study. Quant Imaging Med Surg 2019;9:1014-24.

23. Wang M, Luo Y, Cai H, Xu L, Huang M, Li C, Dong Z, Li ZP, Feng ST. Prediction of type 2 diabetes mellitus using noninvasive MRI quantitation of visceral abdominal adiposity tissue volume. Quant Imaging Med Surg 2019;9:1076-86.

24. Addeman BT, Kutty S, Perkins TG, Soliman AS, Wiens CN, McCurdy CM, Beaton MD, Hegele RA, McKenzie CA. Validation of volumetric and single-slice MRI adipose analysis using a novel fully automated segmentation method. J Magn Reson Imaging 2015;41:233-41.

25. Stocker D, Bashir MR, Kannengiesser SAR, Reiner CS. Accuracy of Automated Liver Contouring, Fat Fraction, and R2* Measurement on Gradient Multiecho Magnetic Resonance Images. J Comput Assist Tomogr 2018;42:697-706.

Cite this article as: Zhang QH, Zhao Y, Tian SF, Xie LH, Chen LH, Chen AL, Wang N, Song QW, Zhang HN, Xie LZ, Shen ZW, Liu AL. Hepatic fat quantification of magnetic resonance imaging whole-liver segmentation for assessing the severity of nonalcoholic fatty liver disease: comparison with a region of interest sampling method. Quant Imaging Med Surg 2021;11(7):2933-2942. doi: 10.21037/qims-20-989 